\title{
The Role of Creativity in Entrepreneurship
}

\author{
Chuyue ZHANG
}

199 Taikang East Road, Ninbo, China, The University of Nottingham Ninbo China

zy13797@nottingham.edu.cn

Keywords: Entrepreneurship, creativity, innovation, competitiveness

\begin{abstract}
This paper evaluates to what extent creativity contributes to entrepreneurship both in theory and in practice. Entrepreneurship is a crucial business conduct in contemporary society, and it is obvious that there is a forceful interaction between creativity and entrepreneurship. The discussion is started from the definition and explanation of entrepreneurship and creativity, and the relationship of them is also addressed. Apart from creativity, other essential elements of entrepreneurship such as innovation, which is decisive to the feasibility of creative idea, are also considered. The paper also includes some personal experience of an Ingenuity program the author participated in as extra evidence for the argument.
\end{abstract}

\section{Introduction}

The modern economy is keeping changing, and to adapt to the new reality, organizations must keep pace with the times. Fillis states that the economy in the present is starting to shift from knowledge based to a new form which is based on creativity, innovation and entrepreneurship, which means that entrepreneurship takes an essential role in the economy today [1]. It is reported by GEM studies that entrepreneurship is one of the main energy of the economy growth of countries [2]. It has provided extensive of job creation and contributed significantly to technological innovation [3]. In terms of individual enterprise, successful entrepreneurship and innovation increase the profitability and sustainability of a company, and thereby improve the competitiveness.

Entrepreneurship has various definitions. It can be viewed as a dynamic process related to opportunity finding, creating and innovating in the marketplace [4]. It can also be an unprecedented combination of existing resources which leads to opportunity for extra profit or benefit [5]. Eisenmann simply identifies entrepreneurship as mobilizing resources over possessed to pursue opportunities [6]. Among all these definitions, creativity is clearly a component of entrepreneurship. It is the derivation of transformation from the former version to a novel product or business [5]. It is about coming up with ingenious ideas or generating things together with an original way. According to Shalley et al., companies can obtain advantage when they identify opportunities in the changing economy circumstance with the assistance of creativity [1].

However, creativity is not the only element related to entrepreneurship. Creativity is the basis of entrepreneurship, but entrepreneurship is more than creativity, it also involves innovation [7]. How to turn creative ideas into innovation and implement them in reality is a problem that entrepreneurs cannot eliminate. This essay will argue that creativity is an essential link to entrepreneurship as it will be beneficial to the development of entrepreneurial enterprise by providing novel ideas, but it cannot work alone without innovation and implementation, which are also important sections for entrepreneurs to achieve their objectives.

\section{The Relationship between Creativity and Entrepreneurship}

Creativity, as is described by Herrmann, is a dynamic activity taken place in the brain consciously or unconsciously to create new ideas. It is a key segment of entrepreneurship [5]. It is indicated by Schumpeter that the creative response to a problem can be identified as the entrepreneurial spirit and the basis of further innovation [8]. Creativity may result in the opening of 
a new business or increase in competitiveness using enhanced product of a current business [9]. Moreover, it can also assist company to discover problems and opportunities in the market constantly. It is an essential trait of entrepreneurs. However, creativity does not merely belong to entrepreneur who is genius. Lumsdaine and Binks criticize that most people have the nature to think creatively, but the external influence of culture impede people's creativity [5]. Therefore, to be creative, people need to break through the barriers and surpass the traditional concept which they believed before.

Creativity is a process leads to change of one historical version of a domain in the culture [5]. The evolution of an item usually involves changing. It is about destroying traditional rules or ideas and finding out new ideas to drive the development, and the difficulty of this process is to realize the paradigm shift [8]. It is claimed by Schermer that the entrepreneurs are someone who break the thinking set and challenge the existing assumptions actuated by their creativity or imagination [8]. They have the ability to discover and exploit opportunities, conceive creative ideas and implement them in the business. This can be seen as evidence that creativity is an essential driving force to entrepreneurship.

To be a successful entrepreneur, it is significant to think creatively. One excellent imaginative idea cannot support the entrepreneur to be successful for long, long-term success belongs to entrepreneurs who are able to put forward creative solutions continually [4]. Utilizing this thinking method, entrepreneurs can produce a completely new product or increase the productivity and efficiency of an existing business, and either way will lead to them be more competitive in the market [7]. Furthermore, creative thinking is also beneficial for entrepreneurs to formulate the whole business strategy. To conduct a business smoothly, it is essential to draw up a comprehensive plan. The business strategy contains numerous aspects such as the resources allocation, the approach, the process and the positioning. All of these can be figured out more efficiently with creative thinking.

When working for the Ingenuity project, the creative thinking was the key for the author to figure most of things out as it allowed the author to think in a new way. Creativity needs to be kept all along and new ideas on the product improving were put forward constantly to ensure the product is competitive enough against other group.

In addition,due to the shift of the economic environment mentioned in the beginning, creativity becomes vital to organizations that are eager to follow the trend. New patterns are required to fit in the changed market[7]. It is indicated by Ko and Butler that creativity and entrepreneurial activity is now being emphasized by a multitude of countries [9]. Developing countries are not merely satisfied with their position as component manufacturers, and they encourage the emergence of entrepreneurial companies within the country.

There are several external factors that can inspire and support creativity. For instance, expertise can cause an influence on creativity. As is demonstrated by Ward, creative ideas entrepreneurs produced do not flashed into their mind, most of them are based on the existing knowledge of the person [10]. Background knowledge needs to be used to consummate and add details to the idea. The author felt the necessity of expertise in the experience of the Ingenuity project. Our group is stuck in the product design section because we do not know whether our idea is able to be supported by current technology, which is caused by the lack of related knowledge. Expertise can be used on opportunities and new emerging trends identification as well as product improving [11]. Social network is another factor which can affect creativity. Generating information from social network is an excellent approach to improve business. People outside the business are more likely to present ideas in relation to aspects have not been covered by the business owner [9]. This type of information will have a positive effect for entrepreneurs on finding out the weakness and novel ideas. This statement is also proved in the Ingenuity project. Feedbacks and suggestions from other groups do facilitate our work to become mature.

Creativity and entrepreneurship are interacting with each other. Researches show that similar to the influence creativity has on entrepreneurship, entrepreneurial behaviour will also affect creativity to some extent. According to Fillis, entrepreneurial management has an effect on creativity [1]. By 
means of changing the work environment, the creativity can be inspired. An example of this is Google, who decorates their company with a colourful design to encourage workers to create. There is another argument that small entrepreneurial enterprise is more likely to innovative than a large one [7]. Flexible work condition and strong group cohesiveness allows both individuals and groups to be creative.

\section{Creativity to Innovation}

In contrast to creativity, innovation is about new value creating. It is an imperative connection between novel ideas and implementations. If creativity is an ability to think new things, then innovation can be described as an ability to do new things [4]. It is demonstrated by Okpara that innovation cannot be achieved without creativity, whereas creativity itself is not sufficient for enterprise to develop or even survive [7]. Creativity is the basis of innovation, but in order to stand out in the marketplace, company needs to generate the creative thinking and identify whether it is suitable for the market. They are different stages of entrepreneurship. Take the experience in the Ingenuity project for example, having new ideas is not enough for us to design a product. The feasibility of our ideas must be evaluated with criteria such as market attractiveness and affordability.

Some researchers argue that creativity is useless without latter process. Levitt states creativity is unduly emphasized by people these days [12]. He criticizes that creative thinking in the abstract is relatively easy compared with innovation which is related to reality, but people have the difficulty to distinguish between them. Thinkers struggle to come up with ideas with novelty, and overlook the practical applicability of their ideas in the market at the same time. It should be realized that imagination and conception is much more abundant than implementation. Furthermore, putting forward ideas to a problem does not equal to solving the problem. The idea will not work until it is truly applied [12]. Creativity needs to be organized in a rigorous approach so that it can be implemented successfully. Okpara points out that creative enterprise dedicates itself to create original product but they can barely afford their new product development [7]. Having creative ideas does not mean completing the business, considerable effort is required to transform ideas into practical products or services and to bring benefit for enterprise. Thus, entrepreneurs must identify the current market situation, understand the customer preference and evaluate the creative idea fully and carefully to ensure it can be adapted in the marketplace.

\section{Conclusion}

To conclude, creativity plays an essential role in entrepreneurship. It relates to numerous factors occur during entrepreneurial activity. Entrepreneur with creativity are more capable of identifying problems and opportunities in the market and raising new ideas and products. Creativity also helps enterprise be more competitive and differentiate with others. It is now generally agreed that depending on the current economy trend, creativity of enterprise is transforming from a good point to an imperative [4]. Thus, the role of creativity may be increasingly significant if company wants to survive.

However, the isolated creativity is not sufficient for entrepreneur to success. Creativity of the product is not the guarantee of its marketability and the profit. Perfect idea will not become useful until it is applied to real market. That is the stage where creativity turns into innovation. To achieve this, enterprises need to analyse the ideas carefully and evaluate their feasibility and usefulness. Additionally, it is also necessary for companies to research for the current market situation and the needs of customers to adapt their products better to the marketplace. Creativity and innovation can be described as the heart of entrepreneurship [7]. Neither of these to items can be avoided. Enterprises need to start from creativity and move to innovation to differentiate themselves in the market and reach the profit maximization. 


\section{Reference}

[1] I. Fillis: submitted to Journal of Enterprising Culture (2010).

[2] P. Westhead and M. Wright, Entrepreneurship: A very short introduction, Oxford University, The United Kingdom, 2013.

[3] R. Bednarzik: submitted to Monthly Labor Review (2000).

[4] D. Kirby, Entrepreneurship, McGraw-Hill Education, The United Kindom, 2002.

[5] E. Lumsdaine and M. Binks,Entrepreneurship, from Creativity to Innovation: Effective Thinking Skills for a Changing World, Trafford, Canada, 2007.

[6] T. Eisenmann: submitted to Harvard business review, 2013.

[7] F. Okpara: submitted to Journal of Asia Entrepreneurship and Sustainability, 2007.

[8] P. Kirkham, S. Mosey and M. Binks, Ingenuity in Practice - a Guide for Clear Thinking, The University of Nottingham, The United Kingdom, 2009.

[9] S. Koand J. E. Butler: submitted to Business Horizons, 2007.

[10] T. B. Ward: submitted to Journal of Business Venturing, 2004.

[11] C. Tu and S. Yang: submitted to International Journal of Innovation, Management and Technology, 2013.

[12] T. Levitt: submitted to Harvard Business Review, 1963. 